\title{
Kleinkinder und ihre Familien zu Zeiten der COVID-19 Pandemie in der Schweiz
}

\author{
Patricia Lannen, Isabelle Duss, Clara Bombach, Nina Graf, Heidi Simoni \\ Marie Meierhofer Institut für das Kind, \\ assoziiertes Institut der Universität Zürich
}

\begin{abstract}
Zusammenfassung: Um Auswirkungen der Einschränkungen zur Eindämmung der COVID-19Pandemie im Frühling 2020 auf den Alltag und das Wohlbefinden von Kleinkindern und ihren Familien zu erfassen, wurden während dieser Zeit in der Schweiz 55 leitfadengestützte Telefoninterviews mit Müttern und Vätern mit mindestens einem Kind im Alter von bis zu 4 Jahren geführt. Die Organisation von Betreuung und gleichzeitigem Homeoffice war anspruchsvoll. Die Eltern berichteten, dass sich für ihr Wohlbefinden und somit auch für dasjenige der Kinder ein strukturierter, aber nicht starrer Tagesablauf, einschließlich flexibler Homeoffice-Lösungen, bewährte. Wichtig waren zudem eine sinnvolle Aufteilung der zusätzlich anfallenden Aufgaben und die Möglichkeit, nach draußen zu gehen. Es werden Vorschläge eruiert, welche aus Sicht der Interviewten zur Entlastung beigetragen hätten.
\end{abstract}

Schlüsselwörter: COVID-19 Pandemie, Kleinkinder, Alltagsgestaltung, Wohlbefinden

\begin{abstract}
Young children and their families during the COVID-19 pandemic in Switzerland Summary: To assess the impact of measures implemented to contain the spread of the COVID-19 pandemic in the spring of 2020 on the daily lives and well-being of young children and their families, 55 semi-structured telephone interviews were conducted with parents of children aged up to 4 years in Switzerland. The logistics and appropriate care of children while simultaneously working at home was reported to be a great challenge. Parents reported that a structured but not rigid daily routine, a sensible division of additional tasks, and the opportunity to go outside proved to be beneficial for their well-being and thus also for that of the children. Parents discuss suggestions that would have helped relieve the burden during that time.
\end{abstract}

Keywords: COVID-19 pandemic, infants, daily routines, well-being

\section{Einleitung}

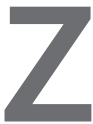
ur Eindämmung der Ausbreitung der COVID-19-Pandemie wurde in der Schweiz ab Mitte März 2020 das öffentliche Leben für zwei Monate stark eingeschränkt und die Landesgrenzen weitgehend geschlossen. Auch Schulen und öffentlich zugängliche Einrichtungen wie Museen, Zoos, Kinos, Hallenbäder, Restaurants sowie alle Geschäfte, die für die Grundversorgung nicht relevant sind, wurden geschlossen. Zudem wurden alle öffentlichen und privaten Veranstaltungen sowie Menschenansammlungen von mehr als fünf Personen verboten. Als weitere Maßnahme wurden alle Arbeitnehmenden aufgefordert, wenn immer möglich im Homeoffice zu arbeiten (Schweizerischer Bundesrat 2020 b). Die Bevölkerung wurde gebeten, zu Hause zu bleiben und die sozialen Kontakte stark einzuschränken.

\section{Situation der Kleinkinder}

Die angeordneten Maßnahmen betrafen in besonderer Weise auch Kleinkinder und ihre Familien. In der Deutschschweiz werden 34\% der Kinder in einer Kinderkrippe ${ }^{1}$ und $49 \%$ der Kin- 
der durch Personen aus dem Umfeld und dabei vor allem von den Großeltern betreut (bei ca. $16 \%$ der Kinder finden beide Betreuungsvarianten gleichzeitig statt) (Bundesamt für Statistik 2019, 2020 b). Kinderkrippen wurden zur Eindämmung der Pandemie während der Zeit der besonderen Maßnahmen entweder ganz geschlossen oder waren nur noch für Kinder von Eltern, welche in der Grundversorgung arbeiten, zugänglich (Eidgenössische Kommission für Familienfragen EKFF 2020). Des Weiteren empfahl der Bundesrat zum Schutz der besonders gefährdeten Personen (Personen ab 65 Jahren und Personen mit bestimmten Vorerkrankungen) dringend, eine Durchmischung der Generationen und somit die Betreuung von Kindern durch die Großeltern zu vermeiden (Schweizerischer Bundesrat 2020 b). Als Konsequenz verschob sich in dieser Zeit die sonst durch Kinderkrippen und Großeltern geleistete Betreuung der Kinder größtenteils auf die Eltern(teile).

Somit verbrachten mehr Kleinkinder als sonst viel Zeit zu Hause mit ihren Eltern, die teils Familienarbeit und Homeoffice vereinbaren mussten. Andere sahen ihre Eltern wenig, wenn diese aufgrund ihres Berufes (z. B. in der Gesundheitsoder Lebensmittelversorgung) sehr beansprucht waren. Die Lebensräume der Kleinkinder, ihr Radius, ihre sozialen Kontakte mit gleichaltrigen Kindern und Familienmitgliedern wurden durch den (teilweisen) Verlust des Zugangs zu wichtigen Orten wie Spielplätzen, Kinderkrippen, Spielgruppen oder von nicht länger möglichen Kontakten zu engen Freunden stark begrenzt.

Brunner et al. (2020a) beschreiben, dass durch die Veränderung der äußeren Strukturen wie zum Beispiel den Wegfall der Kinderkrippen nun die Eltern und die weiteren engen Bezugspersonen gefordert waren, um den Kindern eine Tagesstruktur zu schaffen, die dem Vertrauten möglichst nahe kam (Brunner et al. 2020 a). Brunner et al. (2020 a) betonen, wie wichtig es ist, dass die Eltern dazu animiert werden, eine Tagesstruktur mit einer ausgewogenen Abwechs- lung an Bewegung, Ruhe und Aktivität aufrechtzuerhalten. Dabei sei es zentral, mit den Kindern nach draußen zu gehen (was in der Schweiz auch während der besonderen Maßnahmen noch möglich war), um den Kindern weiterhin wichtige Erfahrungen zu ermöglichen und auch damit die Kinder am Abend müde sind.

\section{Aktuelle Studien zu Auswirkungen von besonderen Maßnahmen}

Aktuelle Studien beschreiben die Auswirkungen von Maßnahmen zur Eindämmung der COVID19-Pandemie vor allem mit einem Fokus auf Erwachsene. So können Trennung von wichtigen Bezugspersonen und Verlust von Freiheiten zu negativen Auswirkungen wie zum Beispiel depressiven Symptomen oder schlechter Schlafqualität führen (Brooks et al. 2020, Huang und Zhao 2020, Rajkumar 2020, Wang et al. 2020). Auch Existenzängste und die Angst selbst am Virus zu erkranken, können bei Erwachsenen zu psychischen Belastungen führen (Bartholomäus und Schilbach 2020). Diese Auswirkungen sind für Eltern von jungen Kindern ebenfalls relevant.

Kürzlich erschienene Fachartikel, welche sich allgemein mit der veränderten Alltagsgestaltung während der COVID-19-Pandemie auseinandergesetzt haben, dokumentieren, welche Herausforderungen sich durch die Maßnahmen für Familien mit Kindern etwa in der Betreuungssituation durch die Schließung der Kinderkrippen ergaben (Wesemann et al. 2020). Dabei wurden Veränderungen der täglichen Routinen und Verluste von sozialen Beziehungen beschrieben.

So fehlten den Kleinkindern die Großeltern oder die Betreuungsperson in der Kinderkrippe (Dalton et al. 2020). Eine Studie aus China zeigte, dass Maßnahmen wie Schulschließungen und Quarantäne bei Kindern zwischen drei und 18 Jahren zu Anhänglichkeit, Unaufmerksamkeit, Reizbarkeit, Schlafproblemen und Alpträumen führen kann (Jiao et al. 2020). 
Studien dazu, wie sich die Situation konkret auf die Kinder und im Speziellen auf Kleinkinder auswirkt, sind allerdings noch rar (Rajkumar 2020). Publizierte Studien zu Auswirkungen von Maßnahmen beziehen sich zudem jeweils auf einen konkreten lokalen Kontext und lokale Maßnahmen. Es muss davon ausgegangen werden, dass das Erleben und die Auswirkungen der Maßnahmen spezifisch sind für die lokal gegebenen Einschränkungen sowie die Dynamik der Pandemie. So wurde zum Beispiel in der Schweiz im Frühling 2020 kein Ausgehverbot ausgesprochen und es mussten sich nur vereinzelte Personen in Quarantäne beziehungsweise Isolation begeben. Viele der international publizierten Studien sind somit für den schweizerischen Kontext nur bedingt aussagekräftig.

\section{Aktuelle Studie}

Mit dem Ziel, sich einen ersten Überblick über das Wohlbefinden von Familien und Kleinkindern zu Zeiten der Maßnahmen zur Eindämmung der Pandemie zu verschaffen, wurde vom Marie Meierhofer Institut für das Kind, ein assoziiertes Institut der Universität Zürich und Kompetenzzentrum für die frühe Kindheit, während des Lockdowns eine Studie umgesetzt. Im Zeitraum zwischen Anfang April und Ende Mai 2020 wurden der Alltag und das Befinden von jungen Kindern bis 4 Jahren untersucht. Dabei standen die folgenden Fragestellungen im Zentrum:

- Wie sieht der Alltag junger Kinder und ihrer Familien aus?

- Wie geht es den jungen Kindern und ihren Familien?

- Was hilft den jungen Kindern und ihren Familien in der gegebenen Situation?

Aus dieser Studie werden im vorliegenden Artikel die Ergebnisse präsentiert und diskutiert.

\section{Methoden}

\section{Studiendesign und Rekrutierung}

Die Studie zielte darauf ab, ein breites Spektrum an Lebenssituationen zu dokumentieren. Die Studie sollte rasch auf die aktuellen Entwicklungen in der Schweiz im Rahmen der Bekämpfung der COVID-19-Pandemie und damit der Maßnahmen, welche die Familien und Kleinkinder direkt betrafen, reagieren. Eine zeitnahe Umsetzung des Studienvorhabens musste gewährleistet werden, um der unerwarteten, einmaligen und besonderen Situation und damit den antizipierten Herausforderungen der Familien in der Bewältigung der Situation Rechnung zu tragen. Es wurde hierfür eine Telefon-Interviewreihe mit Eltern(teilen) von Kleinkindern (bis 4-jährig) realisiert. Die Studienteilnehmenden wurden mithilfe des Schneeballprinzips im beruflichen Kontext und Netzwerk des Marie Meierhofer Instituts für das Kind und vereinzelt auch über das private Netzwerk der Autorinnen angefragt. Dabei wurden insbesondere auch professionelle Unterstützungseinrichtungen wie Mutter-Kind-Zentren und Asylunterkünfte berücksichtigt. Im Rahmen der Interviews wurde eine möglichst große Vielfalt von Lebensumständen abgedeckt, um die Bedeutung der Maßnahmen für unterschiedliche Familien- und Betreuungsarrangements zu erfassen.

\section{Studienteilnehmende}

An der Studie nahmen 47 Mütter und elf Väter mit mindestens einem Kind bis 4-jährig, die im vorliegenden Text als Kleinkinder bezeichnet werden, teil. Bei drei Familien nahmen sowohl Mutter als auch Vater teil, deshalb beziehen sich die Angaben zu den Studienteilnehmenden auf 55 Familien. Die Studienteilnehmenden konnten aus elf verschiedenen Deutsch-Schweizer Kantonen ${ }^{2}$ gewonnen werden. 
$76 \%$ der Familien besaßen eine Schweizer Nationalität und in $78 \%$ der Haushalte wurde deutsch gesprochen. $45 \%$ der Mütter hatten mindestens einen Fachhochschulabschluss. $15 \%$ der Mütter waren alleinerziehend und $20 \%$ der Familien lebten mit geringen sozioökonomischen Ressourcen und waren zum Beispiel von Armut und/oder Arbeitslosigkeit betroffen oder lebten mit Asylstatus. Ein Vergleich der sozio-demografischen Angaben mit der schweizerischen Gesamtbevölkerung ist in Abbildung 1 ersichtlich. Dabei sind geringfügige Unterschiede v. a. im höchsten Bildungsabschluss der Mutter sowie bei Deutsch als Familiensprache zu erkennen. So sind die Mütter in der vorliegenden Studie etwas höher gebildet als in der schweizerischen Gesamtbevölkerung. Die Familien in der vorliegenden Studie gaben hingegen weniger häufig an, dass Deutsch ihre Familiensprache sei, als dies bei der schweizerischen Gesamtbevölkerung der Fall ist.
Im Median hatten die Familien zwei Kinder (Minimum ein Kind, Maximum fünf Kinder) wovon pro Familie ein Kind unter vier Jahre alt (Minimum ein Kind, Maximum drei Kinder) war. Die Kinder unter vier Jahren waren im Durchschnitt 24 Monate alt (Minimum 1 Woche, Maximum 48 Monate). Kurz vor oder während der außerordentlichen Maßnahmen kamen drei Kinder zur Welt und vier der interviewten Frauen waren schwanger.

Fünf Familien gaben an, dass ihr Kind bei einer Infektion ein erhöhtes Risiko für einen schweren COVID-19-Verlauf aufwies. ${ }^{3}$ In sieben weiteren Familien gehörte ein Elternteil zur Risikogruppe (u.a. Diabetes, Asthma, Herzkrankheit).

Vor den Maßnahmen besuchte/n das Kind/die Kinder in $45 \%$ der Familien eine Kinderkrippe, in zwei Familien wurden die Kinder von einer Tagesmutter betreut und in zwei Familien besuchten die Kinder eine Spielgruppe. Zudem

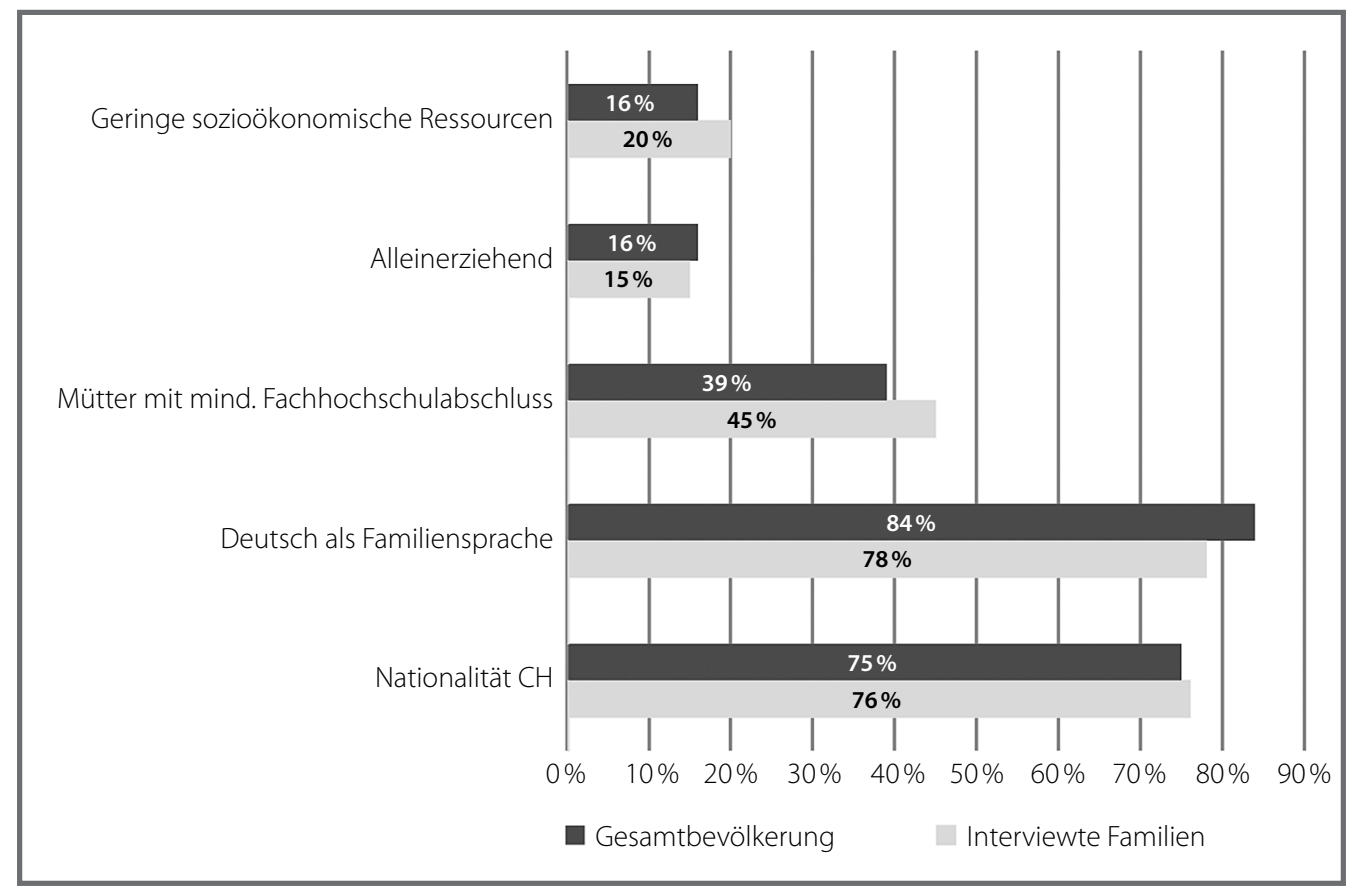

Abb. 1: Vergleich der sozio-demografischen Daten zwischen den interviewten Familien und der schweizerischen Gesamtbevölkerung (Bundesamt für Statistik 2020 a, 2020 c, 2021 a, 2021 b, 2021 c). 
übernahmen die Großeltern in ebenfalls $45 \%$ der Familien regelmäßig die Kinderbetreuung. In neun Familien wurden die Kinder sowohl von den Großeltern als auch von einer Kinderkrippe betreut.

\section{Datenerhebung}

Im Rahmen der Studie wurden durch die Autorinnen dieses Beitrages Telefoninterviews mit Müttern und Vätern durchgeführt. Die 55 Interviews fanden zwischen April und Mitte Mai 2020 mittels eines halb-strukturierten Interviewleitfadens statt. Die Gespräche dauerten mehrheitlich zwischen zehn und 20 Minuten und fanden größtenteils auf Schweizerdeutsch oder Deutsch statt (eines auf Französisch, drei auf Englisch, eines je auf Arabisch und Tigrinya mit Dolmetscher).

Die Interviews umfassten Fragen zum Wohlbefinden der Eltern und Kinder, ihrem Lebensalltag und Ritualen seit Pandemiebeginn, zu Veränderungen aufgrund der Maßnahmen sowie zu Herausforderungen und Chancen dieser Zeit. Zudem wurde nach Art und Weise der Betreuung aktuell sowie vor der Pandemie gefragt wie auch danach, was den Familien aktuell hilft oder sie entlasten würde.

\section{Auswertungsmethoden}

Alle Interviews wurden mithilfe eines AudioAufnahmegeräts festgehalten und auf Deutsch zusammenfassend verschriftlicht. Nach der Verschriftlichung wurden die Audios aus Datenschutzgründen gelöscht. Für die Analyse der Interviews wurden zwei Verfahren aufeinander aufbauend umgesetzt. In einem ersten Schritt wurden in Form eines sequenziellen und rekonstruktiven Analyseverfahrens (Rosenthal 2015) Hypothesen aus dem Datenmaterial an kontrastiven Fallvignetten herausgearbeitet. Das Forschungsteam setzte sich aus verschiedenen wissenschaftlichen Disziplinen zusammen (Entwicklungspsychologie, Erziehungswissenschaften und Sozial- und Kulturanthropologie), was der analytischen Arbeit und Hypothesenbildung sehr zuträglich war.

Anschließend wurden alle weiteren Interviews mithilfe von MAXQDA Version 2018, einer Software zur Analyse von qualitativen Daten, codiert und inhaltsanalytisch nach Mayring (2015) ausgewertet.

\section{Resultate}

Im Folgenden werden die Ergebnisse entlang den Fragestellungen referiert.

\section{Wie sehen der Alltag und die Lebenswelten der jungen Kinder und ihrer Familien aus?}

Die Mehrheit der Familien berichtete, dass sich nach einer Anlaufphase ein Alltag mit einer angepassten Tagesstruktur einpendelte. Dazu führten die Familien teils neue Rituale und Abläufe ein. Wenn dies nicht gelang, war die Familie eher belastet und es kam in einzelnen Familien zum Beispiel zu Schlafproblemen des Kindes.

"Meine Tochter kann nicht gut schlafen. Es ist zum Beispiel 21 Uhr und sie kann nur eine Stunde schlafen und dann erwacht sie um 22 Uhr wieder. Oder sie erwacht um 23 Uhr und bleibt bis vier Uhr früh auf." (Mutter von 17-monatigem Kind).

Eltern, die berichteten, durch das Arbeiten von zu Hause aus stärker als zuvor gefordert zu sein oder neben der Betreuung des Kleinkindes noch ältere Geschwister zu Hause im Homeschooling zu unterrichten, hatten den Eindruck, dass die Bedürfnisse der Kleinkinder oft zurückgestellt werden mussten. Manche Eltern beschrieben ihre Kleinkinder dann als störend, beschäftigungshungriger, anhänglicher und bedürftiger. 
„Man merkt es dem Kleinsten schon an, er reagiert stark. ... Es kommt in den letzten Tagen häufiger vor, dass er quengelt oder , am Hosenbein hängt'." (Mutter von einem Kleinkind und zwei Schulkindern).

Allgemein verlagerte sich durch die Maßnahmen der Fokus der sozialen Kontakte auf den engsten Familienkreis. So berichteten viele Eltern, dass die fehlende Zeit mit gleichaltrigen SpielkameradInnen durch Brüder/Schwestern kompensiert wurde. Geschwisterbeziehungen intensivierten sich dadurch in vielen Familien und gewannen an Wichtigkeit.

„Wir haben gemerkt, dass die Geschwister mehr Zeit zusammen verbringen. Sie spielen sehr schön miteinander. So profitieren sie eigentlich gegenseitig sehr von dieser Situation. Wir haben das Gefühl, dass es ihnen gut tut." (Vater von Kleinund Schulkind).

Dennoch war es für einige Kleinkinder ungewohnt, dass nun die älteren Geschwister vermehrt zu Hause waren und die Zeit mit den Eltern, die sonst alleine verbracht worden wäre, nun geteilt werden musste.

Einige interviewte Eltern berichteten, dass das Social Distancing den Kleinkindern sehr schwer zu vermitteln war.

„Wenn wir spazieren und andere Kinder auf uns zukommen, dann versuchen wir alles, um den Kontakt zwischen ihnen und unseren Kindern zu vermeiden. Das ist schwierig, weil wir dies mit unseren Kindern noch nicht thematisieren können. Plötzlich werden die anderen Kinder als, böse' angesehen. Natürlich sind sie das nicht. Doch ich frage mich schon, was es mit meinem Kind anstellt, wenn wir es immer von anderen Kindern wegziehen müssen." (Elternpaar von zwei Kleinkindern).

Die Veränderungen in der Gestaltung der sozialen Kontakte realisierten bereits die jüngsten Kinder. Aussagen bezüglich der fehlenden so- zialen Kontakte ihrer Kleinkinder zu anderen Kindern außerhalb der Familie stammen vor allem auch von Eltern mit Einzelkindern.

„Wenn Kinder auf dem Spielplatz sind und sie (die Tochter) das sieht, dann schaut sie sehr lange zu den Kindern und zeigt auf diese... Also ich glaube schon, dass ihr das ein wenig fehlt." (Mutter von einem 16-monatigen Kind).

"Mein Kind fragt mich sehr oft, wann es endlich die anderen Kinder wieder sehen und mit ihnen spielen kann. Und wenn wir andere Kinder im Dorf sehen, dann schaut sie ihnen sehr lange und sehr genau aus der Ferne zu." (Mutter von 2 1⁄2-jährigem Kind).

Viele Eltern ganz junger Kinder (bis etwa $1 \frac{1}{2}$ Jahren) berichteten, dass aus ihrer Sicht der eingeschränkte Lebensradius zum Beispiel durch den Wegfall von Spielplatz- oder Zoobesuchen für ihr Kind kaum wahrnehmbar war. Etwas ältere Kinder nahmen den eingeschränkten Lebensradius viel eher wahr und verbalisierten, dass innen zum Beispiel der Spielplatz oder die Kinderkrippe fehle.

„Mein Kind fragt mich jeden Tag, wann sie wieder in die Spielgruppe gehen kann.... Ich glaube, dass siees sehrvermisst." (Muttervon 3-jährigem Kind).

Einige Familien äußerten sich auch zur Dauer der Pandemie. Sie meinten grundsätzlich, dass sie die Maßnahmen zwar im Moment gut umsetzen könnten. Es sei jedoch eine Herausforderung, nicht zu wissen, wie lange die Situation noch andauere. Einige Eltern zeigten sich verunsichert, was die Maßnahmen für die Entwicklung der Kinder bedeuten.

„Wenn es dann länger dauert, finde ich es ein bisschen schwierig für die Entwicklung der Kinder, weil es immer heißt, man soll Abstand halten, nicht zu anderen hingehen. Was bedeutet dies dann für die Entwicklung der Kinder?" (Vater von 21⁄2-jährigem Kind). 
Einzelne Eltern äußerten in den Interviews, dass ihre Kinder in dieser Zeit weniger krank waren als sonst, da sie sich aufgrund der Maßnahmen auch weniger mit anderen Krankheiten ansteckten. Dies betonten vor allem Familien mit einem Kind mit einem erhöhten Risiko zu einem schweren COVID-19-Verlauf.

\section{"Unserem Kind geht es im Moment sehr gut, so gut} wie schon lange nicht mehr, oder eigentlich noch gar nie. Dadurch, dass wir die letzten Wochen bis Monate ziemlich isoliert waren, konnte unser Kind sich nirgendwo anstecken, auch nicht mit anderen Viren. So lange am Stück war es noch nie gesund." (Mutter von Kleinkind mit erhöhtem Risiko zu einem schweren COVID-19-Verlauf).

Grundsätzlich schränkten sich Familien mit einem Kind mit einem erhöhten Risiko zu einem schweren COVID-19-Verlauf stärker ein als andere Familien. Hingegen konnte festgestellt werden, dass unabhängig davon, ob ein Elternteil zur Risikogruppe gehörte oder nicht, sich weder die Sorge über eine eigene Ansteckung noch die daraus folgenden Einschränkungen im familiären Alltag systematisch unterschieden.

Kurz vor oder während den besonderen Maßnahmen zur Eindämmung der Pandemie kamen drei Kinder zur Welt, deren Eltern an der Studie teilnahmen. In dieser Zeit auf sich allein gestellt zu sein, da die Unterstützung zum Beispiel durch die Großeltern wegfiel, erlebten die Familien als große Herausforderung. Hingegen war es für viele sehr entlastend, dass der Partner zum Beispiel durch Kurzarbeit oder Homeoffice mehr zu Hause war. ${ }^{4}$ Die drei interviewten Eltern berichteten auch, dass sie es bedauerten, ihren Familienzuwachs nicht rasch nach der Geburt Freunden und Verwandten vorstellen und damit ihre große Freude teilen zu können.

„Er (mein Mann) konnte mir kurz vor und auch nach der Geburt viel helfen. Das Homeoffice brachte in dieser Hinsicht viele Vorteile." (Mutter von Neugeborenem und 1 1 12 -jährigem Kind).

\section{Wie geht es den jungen Kindern und den Familien?}

Die Interviewten beschrieben die Arbeit im Homeoffice mit Kleinkindern sehr unterschiedlich. Grundsätzlich wurde die Vereinbarkeit von Erwerbsarbeit und Kinderbetreuung als äußerst anspruchsvoll beschrieben. Entscheidend für ein gutes Gelingen war die Möglichkeit, in der Wohnung einen ruhigen Arbeitsplatz einzurichten bzw. einrichten zu können.

Die Eltern berichteten, dass es für kleine Kinder oft nur schwer verständlich war, warum die Erwachsenen zwar zu Hause, aber gleichzeitig nicht verfügbar waren.

„Es ist sehr schwierig im Homeoffice mit einem kleinen Kind zu arbeiten. Denn er sieht uns so viel und weiß, dass wir da sind. Für ihn ist es schwierig zu verstehen, warum er Mami und Papi beim Arbeiten nicht stören darf. Dies ist für ihn neu und er kannte das bis jetzt noch nicht. Denn wenn wir sonst zu Hause waren, waren wir für ihn da." (Vater von 1 1 1/2-jährigem Kind).

Für das Befinden der Familie war des Weiteren zentral, inwiefern die Arbeitszeiten flexibilisiert werden konnten, um ein gleichzeitiges Arbeiten und Betreuen der Kinder zu umgehen. So versuchten viele Eltern Arbeitsstunden am Abend, in der Nacht, an den Wochenenden oder während der Schlafenszeiten der Kinder nachzuholen. Wenn dies nicht möglich war, d.h. wenn gleichzeitiges Betreuen und Arbeiten nötig war, stieg die Belastung in den Familien erheblich. Gleichzeitig kam es durch die Flexibilisierung aber auch zu einer Vermischung von Arbeit und Freizeit.

Es zeigte sich, dass die Familien von den Arbeitgebern der Mütter mehr Flexibilität erwarteten als von den Arbeitgebern der Väter.

Zudem wurde berichtet, dass Familien, denen es gelang die zusätzlich anfallenden Aufgaben wie Haushalt, Einkaufen, Betreuung der Kinder 
oder Homeschooling auf beide Elternteile zu verteilen, den Alltag als weniger belastend wahrnahmen. Dies zeigte sich unabhängig davon, ob beide Elternteile hochprozentig oder nur ein Elternteil erwerbstätig war. Wenn die zusätzlichen Aufgaben eher auf eine Person fielen, was häufig auf die Mütter zutraf, stieg bei diesen die Belastung.

Insbesondere alleinerziehende Mütter berichteten, dass sie sich durch den Wegfall der externen Kinderbetreuung gestresst fühlten. Sie spürten die Kumulierung der zusätzlich anfallenden Arbeiten besonders stark.

"Für mich ist es sehr anstrengend, das Kind zu betreuen und gleichzeitig eine Online-Weiterbildung zu absolvieren. Auch die Stellensuche inklusive Bewerbungen schreiben ist dadurch sehr kompliziert." (Alleinerziehende Mutter von 17-monatigem Kind).

Die Möglichkeit, die Strukturen des Alltags etwas zu flexibilisieren, brachte bei vielen Familien, vor allem wenn beide Eltern erwerbstätig waren, eine Entlastung. So war es möglich, auch tagsüber mehr Zeit mit den Kindern zu verbringen. Ein Teil der Eltern berichtete, dass ihnen dies ermöglichte, besser auf die Bedürfnisse der Kleinkinder einzugehen. Durch die zusätzliche Familienzeit entstand oft ein Gefühl des Zusammenwachsens der Familienmitglieder. Dabei wussten es die Interviewten besonders zu schätzen, dass die Mahlzeiten gemeinsam eingenommen werden konnten. Gerade die voll berufstätigen Eltern, häufig die Väter, erhielten mehr Einblick in den und Beteiligung am Familienalltag.

„So können wir auch gemeinsam Zeit während des Mittagessens verbringen und bekommen mit, was unser Kind während des Tages erlebt, was er sagt, was er macht." (Elternpaar von einem $1 \frac{1}{2}$-jährigen Kind).

"Ich glaube, mein Mann hat nun ein wenig mehr Verständnis dafür, wie der Alltag mit kleinen Kindern ist. Er kannte das ja vorher auch schon, jedoch nicht in diesem Ausmaß wie jetzt. Das hat durchaus einen positiven Effekt." (Mutter von zwei Kleinkindern).

„Es ist eigentlich schön, dass wir jetzt so viel Zeit haben zu dritt. Ich glaub mein Kind schätzt das auch, dass er (der Vater) so viel da ist." (Mutter von $1 \frac{1}{2}$-jährigem Kind).

"Er (der Vater) hat viel intensivere Zeiten mit unserem Kind und das genießen die beiden auch sehr." (Mutter von 6-monatigem Kind).

Die intensivierte Nähe führte bei einzelnen Familien aber auch zu Gefühlen der Überforderung und Erschöpfung. Dies kam gehäuft bei Familien vor, die in beengten räumlichen Verhältnissen lebten und/oder in dieser Zeit ganz auf sich alleine gestellt waren.

"ICh habe keine Energie, um mit den Kindern zu spielen". (Alleinerziehende Mutter mit einem Kleinkind und zwei Schulkindern).

"Natürlich ist es auch sehr anstrengend, den ganzen Tag mit dem Kind zu verbringen. Da ich mich im Moment nicht von meiner Tochter erholen kann, staut sich alles ein bisschen auf und es ist so viel schwieriger ruhig zu bleiben und gegenüber meiner Tochter nicht laut zu werden". (Alleinerziehende Mutter von 2 12 -jährigem Kind).

Einige Eltern berichteten, dass die Situation bei ihnen zu mehr Paarkonflikten führte.

"Negativ ist natürlich, dass man so eng aufeinanderhockt ... die Reibungen sind natürlich schon ein wenig größer, das Streitpotenzial ist ein wenig höher, weil es einfach auch mehr Dinge gibt, über die man diskutieren kann." (Mutter von 16-monatigem Kind).

Einige Interviewte berichteten von einem Eindruck, dass sie durch diesen intensiven Kontakt nun besser als Eltern im Team funktionierten als zuvor. 
Für Familien mit geringen sozio-ökonomischen Ressourcen zeigte sich bezüglich der empfundenen Belastung, dass es zentral war, wie gut sie in Unterstützungsangebote eingebunden waren. Familien, die in dieser Zeit institutionell unterstützt waren (z. B. durch eine Mutter-KindInstitution), ging es tendenziell besser als alleine lebenden, vorbelasteten Familien. Sie berichteten von einem Gefühl der Sicherheit, dass immer jemand für sie da sei und sie sich dadurch auch nicht auf sich allein gestellt fühlten. In denjenigen Familien, in denen das nicht der Fall war, nahm ein Gefühl der Isolation und Überforderung zu. Auch fiel es vielen dieser Familien schwer zu formulieren, was ihnen in dieser Situation Entlastung bringen würde.

"Ich bin sehr froh, dass ich im Moment in einer Institution lebe. So habe ich noch Menschen um mich und sitze nicht allein in einer Wohnung. Durch die Institution finde ich auch immer eine Betreuung für mein Kind." (Mutter von 1 1/2-jährigem Kind in Mutter-Kind-Institution).

\section{Was hilft den Familien?}

Die interviewten Eltern berichteten vor allem von Entlastung in dieser Zeit durch innovative Betreuungslösungen, die Aufrechterhaltung eines Minimums an sozialen Kontakten oder durch Auszeiten, die sie sich immer wieder einmal gönnen konnten: Es gab Familien, die sich mit einer befreundeten Familie in erweiterte Isolation begaben und die Kinder abwechselnd betreuten. Oder die Großeltern „betreuten“ die Kinder zeitweise via Videochat:

"Wir haben Dinge gefunden, welche unseren Alltag erleichtern. Also zum Beispiel, hauptsächlich, dass unser Kind häufig über Skype von meinen Eltern beschäftigt wird, aber auch von den Eltern meines Mannes. ... Meine Mutter erzählt den Kindern Geschichten. Das funktioniert. Und mein Vater spielt eben auch Kasperlitheater oder solche Sachen vor." (Elternpaar von zwei Kleinkindern).
Das Thema digitale Medien war in den Interviews oft mit viel Ambivalenz besetzt. Einerseits zeigten sich die Eltern entlastet durch deren Einsatz. Andererseits äußerten sie Verunsicherung darüber, wie sich die Beschäftigung der Kleinkinder mit digitalen Medien auf ihre Entwicklung auswirkt.

"ICh mache mir viele Sorgen, wie optimal das für mein Kind ist, weil es im Moment jetzt recht viele Sendungen schaut und ich weiß halt nicht... es ist ja immer so unterschiedlich, ob das schlimm ist oder nicht, es gibt da so verschiedene Haltungen. Aber es ist mir nicht so wohl. Es ist mir wohler, wenn er in der Kinderkrippe ist." (Mutter von Klein- und Schulkind).

Ganz zentral war es für die Familien, dass sie nach draußen gehen konnten. Sie berichteten, dass sie aufgrund der geschlossenen Spielplätze die Umgebung zu Fuß oder mit dem Fahrrad neu erkundeten und sie dies stark entlastete. Dabei griffen viele Eltern auch auf ganz einfache Aktivitäten zurück.

„Mit kleinen Kindern ist es fast egal, was man draußen macht. Es muss nicht spektakulär sein. Einfach in den Wald gehen und Steine werfen reicht bereits." (Vater von drei Kleinkindern).

Einzelne Familien, die sich stark vor einer Ansteckung mit dem Virus fürchteten, verbrachten mit ihren Kindern jedoch kaum Zeit draußen, was für alle Beteiligten belastend war. So waren die Eltern gefordert, ihre Kinder adäquat zu beschäftigen und die Kinder fühlten sich oft gelangweilt und unzufrieden.

„Wir konzentrieren uns stark auf unsere Kinder und kümmern uns um sie, da wir nicht möchten, dass sie aus der Wohnung gehen. Aber darin bestehen auch unsere Schwierigkeiten und Herausforderungen. So waren unsere Kinder vor der Corona-Krise sehrglücklich und zufrieden. Aber zurzeit fühlen sie sich gelangweilt. Ihre Stimmung hat sich auch verändert. Sie reagieren oft mit Wutanfällen." (Vater von zwei Kleinkindern und drei Schulkindern). 
Eine adäquate Beschäftigung und Förderung für die Kinder zu finden, empfanden einige Eltern zunehmend als große Herausforderung. Deshalb schätzten sie es sehr, dass von extern Ideen und Vorschläge dafür gemacht wurden.

"ICh finde es super, das neue Ideen aufgekommen sind wie zum Beispiel ein Online-Tanzen mit dem Kind. Das finde ich wirklich eine gute Sache." (Mutter von 1-jährigem Kind).

„Wir sind Teil einer WhatsApp-Gruppe, in der zum Beispiel Bastelideen weitergeschickt werden oder was man sonst alles noch machen könnte mit den Kindern." (Mutter von einem Klein- und einem Schulkind).

Eltern erlebten den Alltag mit ihren Kindern entspannter, wenn sie mit ihnen in einem engen Austausch blieben, sich auf sie einließen, ihre Fragen mit ihnen besprachen sowie an ihrer Welt teilnahmen.

Vorhandene oder fehlende Flexibilität beim Homeoffice und das Verständnis der erschwerten Vereinbarkeit von Familie und Erwerbsarbeit durch den Arbeitgeber wurden mehrfach und ausdrücklich als zentrales Element der Entlastung bzw. Belastung betont.

„Entlastend dabei wäre sicher ein besseres Verständnis des Arbeitsgebers und der Gesellschaft allgemein, dass es zurzeit unmöglich ist, dieselbe Arbeit auf demselben Niveau zu leisten wie vorher." (Elternpaar von zwei Kleinkindern).

Auch zu wissen, an wen man sich wenden könnte, falls man notfallmäßig doch eine Betreuung bräuchte, entlastete die Eltern.

Viele Eltern berichteten, dass es Ihnen große Entlastung bereitet hätte, wenn sie ihre Kinder auch nur einen Tag pro Woche in die Kinderkrippe hätten bringen können. Die finanzielle Belastung, die Kinderkrippen-Kosten auch während der besonderen Maßnahmen weiterzutragen, war in den Interviews deutlich spürbar. ${ }^{5}$
„Was ich sehr schätzen würde, wäre, wenn der Bund oder der Kanton sich irgendwie dazu durchringen könnten, die Kosten für die Kinderkrippen, welche ja anfallen, obwohl die Kinder nicht dort sind, zu vergüten." (Mutter von einem Kleinkind).

Einige Eltern merkten an, dass sie sich eine klarere Kommunikation des Bundesrats zur Frage der Gefährlichkeit bzw. Unbedenklichkeit des physischen Kontakts junger Kinder untereinander gewünscht hätten.

"Ich würde mir wünschen, dass die Kommunikation vonseiten des Bundesrates über die Kindersituation besser und klarer verständlich gemacht wird. Es ist nicht klar, ob und wie viele Kinder miteinander spielen dürfen. Den Umgang mit diesen Unklarheiten finde ich schwierig." (Vater mit drei Kleinkindern).

\section{Schlussfolgerungen}

Die Bewältigung der Umstellungen und die Alltagsgestaltung während der Zeit der besonderen Maßnahmen fielen in den verschiedenen Familien sehr unterschiedlich aus. Viele Familien berichteten, dass sich nach einiger Zeit neue Tagesabläufe und Rituale einpendelten. Gemäß Aussagen der Eltern bewährte sich ein strukturierter, aber nicht starrer Tagesablauf. Wenn dies gelang, wurde die größere Durchlässigkeit zwischen verschiedenen Lebensbereichen von vielen Familien durchaus als positiv und als Chance erlebt. Dies ermöglichte vielen Familien zusätzliche gemeinsame Zeit, speziell für vollzeit erwerbstätige Väter. Ähnliche Befunde werden auch in anderen Studien berichtet: So konnten zum Beispiel Weissbourd et al. (2020) zeigen, dass $68 \%$ der befragten Väter sich seit der Pandemie ihren Kindern näher fühlen, 51 \% der Väter ihre Kinder während der Zeit besser kennenlernten oder 54\% der Väter den Gefühlen der Kinder mehr Aufmerksamkeit schenkten. Auch wurde berichtet, dass viele Geschwisterbeziehungen in dieser Zeit belebt und gestärkt wurden. 
Familien, denen es nicht gelang, im Alltag eine neue Struktur zu finden und die Kinder ausreichend und sinnvoll zu beschäftigen, waren eher belastet. Dies zeigte sich in dieser wie auch in anderen Studien (Jiao et al. 2020) zum Beispiel in Schlafschwierigkeiten der Kinder.

Wie stark die Familie belastet war, hing stark davon ab, wie gut es möglich war, die Arbeitszeiten zu flexibilisieren. Gerade Kleinkinder können sich nur kurze Zeit alleine beschäftigen, was ein gleichzeitiges Arbeiten und Betreuen fast unmöglich macht. Die Familie war zudem stärker belastet, wenn die zusätzlichen Aufgaben für den Haushalt und die Kinderbetreuung von einer Person übernommen und nicht auf die Erwachsenen verteilt wurden. Ausschlaggebend dafür war nicht, ob die Mutter erwerbstätig war oder nicht, sondern die Zuordnung von Haushalt und Kinderbetreuung. Wenn sich Eltern eher an einem traditionellen Familienmodell orientierten, übernahmen häufig die Frauen die im Haushalt und in der Kinderbetreuung anfallende Mehrarbeit. Zudem zeigte sich in den Interviews, dass Arbeitgeber/innen der Mütter flexibler auf die Veränderungen durch die Pandemie-Maßnahmen reagierten und Spielräume zuließen, die den Vätern nicht zugestanden wurden. Eine Orientierung an traditionellen Rollenverteilungen bzgl. Haus- und Familienarbeit während Krisen konnte auch in anderen Studien nachgewiesen werden (Bütikofer et al. 2020, Hank und Steinbach 2020, National COVID-19 Science Task Force 2020, Wenham et al. 2020). Dabei ist festzuhalten, dass dies bei den Müttern in der vorliegenden Studie durchaus zu einer erhöhten Belastung führte.

Aus der Interviewreihe wurden hauptsächlich Informationen darüber bekannt, wie es den Eltern mit der neuen Situation ging und welchen Herausforderungen sie durch den neuen Alltag begegneten. Ein Bild über das Leben der Kleinkinder selber und ihr Wohlbefinden ließ sich so nur bruchstückhaft erfassen. Dies lag nicht zuletzt auch an der gewählten Forschungsmethode von Telefon-Interviews mit Eltern, die aufgefordert waren, über ihren Lebensalltag und das Befinden der Kinder zu berichten. Trotzdem fiel auf, dass das Befinden der Kinder weniger thematisiert wurde. Im Vergleich dazu nahm das Empfinden und die Wahrnehmung der Eltern, welche die neue Lebenssituation organisieren mussten und die Einschränkungen ihren Kindern zu vermitteln versuchten, deutlich mehr Raum ein. Wir deuten diese Beobachtung als Ausdruck dessen, dass die Eltern(teile) selber sehr mit der Situation beschäftigt waren und deshalb Mühe hatten, ihren Fokus auf die Befindlichkeiten der Kinder zu richten.

In den Interviews zeigte sich, dass einige der Maßnahmen für die Kleinkinder manchmal nur schwer verständlich waren. Eltern erzählten davon, dass sich ihre Kinder fragten, warum ein Elternteil zwar zu Hause, aber trotzdem nicht verfügbar war. Oder warum es plötzlich nicht mehr möglich war, mit den Großeltern oder anderen Kindern Zeit zu verbringen. Diese Ergebnisse der Studie zeigen eindrücklich, wie wichtig es ist, mit den Kindern in altersgerechter Form über diese Maßnahmen zu sprechen und dabei zum Beispiel dem Kind zu erklären, weshalb es sich einer Person nicht nähern sollte (Brunner et al. 2020 c). Zudem sollte auch Schwieriges angesprochen und die eigene Stimmung als Eltern benannt werden, denn Kinder spüren sehr gut, wenn die Eltern beunruhigt sind (Brunner et al. 2020 b).

Für ihr Wohlbefinden und ihr körperliches, emotionales und soziales Gedeihen und zur Entdeckung der Welt benötigen gerade Säuglinge und Kleinkinder direkte persönliche Kontakte zu wenn immer möglich mehreren nahen Bezugspersonen und zu anderen Kindern (Brunner et al. 2020 c, Shuey 2020, Wustmann Seiler und Simoni 2016) sowie Anregungen über verschiedene Sinne (Fonagy und Target 2002). Im Rahmen der Studie zeigte sich, dass Kinder 
während der Zeit der besonderen Maßnahmen deutlich weniger Kontakte mit anderen Kindern hatten als sonst. Das wurde von den Interviewten für die Kinder dann als besonders herausfordernd beschrieben, wenn die Kinder keine Geschwister hatten. Um gewisse Anregungen und Erfahrungen, die zum Beispiel durch den fehlenden Austausch mit Peers wegfielen, für eine gewisse Zeit zu kompensieren, sind Kleinkinder erst recht darauf angewiesen, dass sie in dieser Zeit durch liebevolle, verlässliche, vertraute und verfügbare Bezugsperson begleitet werden (Dawson et al. 2003, Murray und Cooper 1997, Stein et al. 1991, Wustmann Seiler und Simoni 2016). Bei anhaltendem Stress und Überforderung der Eltern können jedoch auch gute elterliche Kompetenzen zermürbt werden und der Fokus auf die Bedürfnisse der Kinder ist dabei unter Umständen nicht mehr gewährleistet. Bei hoher Belastung der Eltern bestehen somit durchaus Bedenken, dass gewisse Kinder einem erhöhten Risiko für Kindeswohlgefährdungen ausgesetzt sind (The Alliance for Children Protection in Humanitarian Action et al. 2020). Deshalb sollten die Eltern entlastet werden, um die Gesundheit und Entwicklung der Kleinkinder zu schützen und somit kurzwie auch längerfristige negative Folgen zu vermeiden.

Verunsicherung herrschte bei vielen Eltern in Bezug auf den Einsatz digitaler Medien. Einerseits ermöglichten es die digitalen Medien, zum Beispiel mit den Großeltern in Kontakt zu bleiben oder gar, dass die Großeltern sich eine Weile mit den Kindern beschäftigten, um den Eltern Ruhe zur Erholung zu ermöglichen. Andererseits machten sich Eltern auch Sorgen, welchen Einfluss die digitalen Medien auf die Entwicklung ihres Kindes haben könnten. Richtlinien zur sinnvollen Nutzung von digitalen Medien in der frühen Kindheit, die auf belastbaren Forschungsergebnissen aufbauen und die die Eltern in ihrer Haltung und ihrem Tun unterstützen und bestärken, wären hilfreich und empfehlenswert (Sticca et al. 2020).
Die Eltern berichteten von unterschiedlichsten Lösungen, um für sich selbst, aber auch für die Familie Entlastung zu schaffen. So begaben sich verschiedene Familien in eine erweiterte Isolation mit einer anderen Familie aus der direkten Nachbarschaft.

In der Schweiz erwiesen sich wichtige Aspekte der Vereinbarkeit von Familie und Beruf als ausschlaggebend, um die Familie durch diese Zeit zu tragen. Die Systemrelevanz der familienergänzenden Betreuung durch Kinderkrippen und Großeltern zeigte sich in dieser Zeit auf eindrückliche Weise. Dies gilt es, gesamtgesellschaftlich anzuerkennen und entsprechend wertzuschätzen. Und es gibt einen wichtigen Hinweis darauf, welche Ressourcen fehlen, wenn Familien nicht auf Großeltern zurückgreifen können oder ein sehr kleines oder gar kein soziales Netzwerk haben.

Wiederholt wurde von den befragten Familien als zentral betont, dass mit den Kindern Zeit im Freien verbracht werden konnte.

\section{Limitationen}

Die Pandemie und ihre Maßnahmen haben diverse Forschungsfragen hervorgebracht, deren Beantwortung einerseits in einem eng gesteckten Zeitrahmen und zugleich unter methodisch sehr beschränkten Möglichkeiten realisiert wurden. Dies gilt auch für die vorliegende Studie. Nach Teti et al. (2020) sei es auch dank qualitativer Studien möglich, die Auswirkungen des historisch relevanten Ereignisses der COVID-19-Pandemie zu dokumentieren. Diese Feststellung unterstützt unsere Methodenwahl qualitativer Interviews mit Elternteilen. Gleichzeitig muss jedoch betont werden, dass mithilfe der Telefoninterviews das Verhalten der Kinder nur über die Beobachtungen der Eltern(teile), die sich zu einem Gespräch bereit erklärten, dokumen- 
tiert werden konnte. Darüber hinaus handelt es sich in dieser Studie um eine kleine Stichprobe sowie ein hypothesengenerierendes Verfahren, deren Generalisierbarkeit limitiert ist. Erkenntnisse aus dieser Studie, wie zum Beispiel, dass die Flexibilität des Arbeitgebers oder die Arbeitsaufteilung der zusätzlich anfallenden Aufgaben zwischen den Eltern einen großen Teil der Belastung erklären, könnten in Studien mit einer größeren Stichprobe hypothesenprüfend untersucht werden. Die Art der erhobenen Daten sowie die Natur des querschnittlichen Designs lassen keine Schlüsse über die längerfristige Auswirkung auf die Entwicklung der Kinder zu.

\section{Bedeutung für die Praxis}

Um negative Konsequenzen für junge Kinder zu reduzieren, ist es zentral, das Wohlbefinden der Eltern sicherzustellen und mit geeigneten Maßnahmen und Informationen zu unterstützen. Hierfür bewährten sich innerhalb der Familie ein strukturierter, aber nicht starrer Tagesablauf, sinnvolles Aufteilen der zusätzlich anfallenden Aufgaben sowie das Aufstellen eines Notfallplans. Innovative Betreuungslösungen, wie das Eingehen von kleinen, überschaubaren Nachbarschaftsnetzwerken oder die zeitlich begrenzte Kinderbetreuung durch Großeltern via Videochat, waren zudem dienlich und ermöglichten sozialen Austausch sowie zwischendurch dringend notwendige Verschnaufpausen für die Eltern. Mit Blick auf die Arbeitgeber kann die Notwendigkeit einer Ermöglichung zur besseren Vereinbarkeit von Familienleben und Berufstätigkeit nicht genug betont werden. Dies bedeutet, dass in dieser herausfordernden Zeit flexible Lösungen notwendig sind. Gerade auch Familien, die in prekären Verhältnissen leben, gilt es zu identifizieren und zu unterstützen, auch um eine allfällige Isolation oder deren drohende Akzentuierung zu vermeiden.
Zuletzt ist hervorzuheben, dass die Möglichkeit, mit den Kindern und selber nach draußen gehen zu können, für die Familien zentral war. Eine Ausgangssperre hätte die Belastung der Familien wohl erheblich verschärft. Sie sollte im Sinn einer ganzheitlichen Perspektive auf die physische und psychische Gesundheit der Bevölkerung als letzte Maßnahme zur Eindämmung einer Epidemie eingesetzt werden.

\section{Anmerkungen}

1 In der Schweiz beginnt der Kindergarten im Alter zwischen 4 und 5 Jahren.

2 Die Schweiz zählt 26 Kantone.

3 Die Einschätzung für ein erhöhtes Risiko basiert auf Aussagen der Eltern und wurde medizinisch nicht nachgeprüft. Die Kinder litten an verschiedenen genetischen Syndromen mit Herzfehlern in unterschiedlichen Ausprägungen, sowie Lungenerkrankungen.

4 Gesetzeslage zum Vaterschaftsurlaub zum Studienzeitpunkt in der Schweiz: „Das schweizerische Bundesrecht sieht keinen Vaterschaftsurlaub vor. Derzeit kann ein Vater bei der Geburt seines Kindes die üblichen, im Obligationenrecht vorgesehenen freien Tage in Anspruch nehmen, wie etwa bei einem Umzug, einem Todesfall oder wenn er heiratet. Meist haben Väter Anspruch auf einen oder zwei freie Tage. Einzelne Branchen oder Unternehmen gewähren jedoch einen längeren Vaterschaftsurlaub. Die Dauer des Urlaubs und die Entschädigung unterscheiden sich je nach Bereich und Unternehmen. Erwerbstätige Mütter haben Anspruch auf einen bezahlten Mutterschaftsurlaub von 14 Wochen." (Bundesamt für Sozialversicherungen BSV) Am 27. September 2020 wurde in der Schweiz die Einführung eines 14-tägigen Vaterschaftsurlaubes beschlossen.

5 Ende Mai 2020 entschied der Bundesrat, dass die Kosten teilweise durch die öffentliche Hand zurückerstattet werden (Schweizerischer Bundesrat 2020a).

Erstautorin:
Dr. phil. Patricia Lannen
Marie Meierhofer Institut für das Kind
Pfingstweidstr. 16
CH-8005 Zürich




\section{Literatur}

Bartholomäus, M., Schilbach, L. (2020): Psychisch gesund bleiben während Social Distancing, Quarantäne und Ausgangsbeschränkungen auf Grund des Corona-Virus. Verhaltenstherapeutische Interventionen in einem Kurzprogramm zur Selbstanwendung, Max-Planck-Institut für Psychiatrie, München

Brooks, S. K., Webster, R. K., Smith, L. E., Woodland, L., Wessely, S., Greenberg, N., Rubin, G. J. (2020): The psychological impact of quarantine and how to reduce it: rapid review of the evidence. The Lancet 395 (10227), 912 - 920, http://dx.doi.org/10.1016/ S0140-6736(20)30460-8

Brunner, S., Hardegger, K., Salis, G. von, Simoni, H. (2020 a): COVID-19-Pandemie und Beziehungskontinuität für junge Kinder. Überlegungen aus gesundheitlicher und entwicklungspsychologischer Perspektive. In: https://www.mmi.ch/uploads/Down loads/Covid/MMI_Covid_Beziehungskontinuitaet_ DE_200331.pdf, 29.12.2020

Brunner, S., Hardegger, K., Salis, G. von, Simoni, H. (2020 b): Mit jungen Kindern über die COVID-19-Pandemie reden. In: https://www.mmi.ch/uploads/Down loads/Covid/MMI_Covid_Gespraeche_mit_Kindern_ DE_200331.pdf, 29.12.2020

Brunner, S., Gasser-Haas, O., Hardegger, K., Salis, G. von, Simoni, H. (2020 c): Social Distancing. Beziehungsgestaltung mit jungen Kindern in Zeiten der COVID-19 Pandemie. In: https://www.mmi.ch/uploads/ Downloads/Covid/MMI_Covid_Beziehungsgestaltung_ DE_Jun2020.pdf, 29.12.2020

Bundesamt für Sozialversicherungen BSV: Vaterschaftsurlaub: die Vorlage im Detail. In: https://www. bsv.admin.ch/dam/bsv/de/dokumente/eo/faktenblaet ter/hintergrund-vaterschaftsurlaub.pdf.download.pdf/ hintergrund-vaterschaftsurlaub.pdf, 28. 12. 2020

Bundesamt für Statistik (2019): Erhebung zu Familien und Generationen, 2018. Erste Ergebnisse. In: https://www.bfs.admin.ch/bfs/de/home/statistiken/ bevoelkerung/erhebungen/efg.assetdetail.10467788. html, 29.12.2020

Bundesamt für Statistik (2020 a): Bildungsstand der Bevölkerung - Daten des Indikators. In: https://www. bfs.admin.ch/bfs/de/home/statistiken/wirtschaftlichesoziale-situation-bevoelkerung/gleichstellung-fraumann/bildung/bildungsstand.assetdetail.12527185. html, 19.3.2021

Bundesamt für Statistik (2020 b): Kinder unter 13 Jahren, die familienergänzend betreut werden, nach Betreuungsform und Alter, 2018. In: https://www.bfs. admin.ch/bfs/de/home/statistiken/bevoelkerung/ familien/familienergaenzende-kinderbetreuung. assetdetail.12927321.html, 11.3.2021
Bundesamt für Statistik (2020 c): Ständige Wohnbevölkerung nach Alter, Geschlecht und Staatsangehörigkeit, 2010-2019. In: https://www.bfs.admin.ch/ bfs/de/home/statistiken/bevoelkerung/standentwicklung/alter-zivilstand-staatsangehoerigkeit. assetdetail.13707177.html, 19.3.2021

Bundesamt für Statistik (2021 a): Armutsgefährdung ausgewählter Haushaltstypen. In: https://www. bfs.admin.ch/bfs/de/home/statistiken/wirtschaftlichesoziale-situation-bevoelkerung/soziale-situationwohlbefinden-und-armut/armut-und-materielleentbehrungen/armutsgefaehrdung.assetdetail.1534 4726.html, 19.3.2021

Bundesamt für Statistik (2021 b): Einfamilienhaushalte nach Anzahl und Alter der Kinder. In: https://www.bfs.admin.ch/bfs/de/home/statistiken/ kataloge-datenbanken/tabellen.assetdetail.16005644. html, 19.3.2021

Bundesamt für Statistik (2021 c): Hauptsprachen nach Sprachgebiet. In: https://www.bfs.admin.ch/bfs/ de/home/statistiken/bevoelkerung/sprachen-religio nen/sprachen.assetdetail.15384653.html, 19.3.2021

Bütikofer, S., Craviolini, J., Hermann, M., Krähenbühl, D. (2020): Schweizer Familien in der Covid-19Pandemie. Spezialauswertung des SRG-CoronaMonitors zu Familien- und Betreuungsstrukturen im Kontext der Krise, Forschungsstelle sotomo, Zürich

Dalton, L., Rapa, E., Stein, A. (2020): Protecting the psychological health of children through effective communication about COVID-19. The Lancet Child \& Adolescent Health 4 (5), 346-347, http://dx.doi.org/ 10.1016/S2352-4642(20)30097-3

Dawson, G., Ashman, S. B., Panagiotides, H., Hessl, D., SElf, J., Yamada, E., Embry, L. (2003): Preschool outcomes of children of depressed mothers: Role of maternal behavior, contextual risk and children's brain activity. Child development 74 (4), 1158-1175

Eidgenössische Kommission für Familienfragen EKFF (2020): Corona-Krise gefährdet Vorschulbetreuung - Politik und Behörden müssen handeln. In: https://www.ekff.admin.ch/fileadmin/user_upload/ ekff/03stellungnahmen/2020/200402_Corona-Krise_ gefaehrdet_Vorschulbetreuung_Stellungnahme_ EKFF.pdf, 4. 8. 2020

Fonagy, P., Target, M. (2002): Early intervention and the development of self-regulation. Psychoanalytic Inquiry 22 (3), 307 - 335

Hank, K., Steinbach, A. (2020): The virus changed everything, didn't it? Couples' division of housework and childcare before and during the Corona crisis. Journal of Family Research 2020, 1 - 16, http://dx.doi. org/10.20377/jfr-488 
Huang, Y., Zhao, N. (2020): Generalized anxiety disorder, depressive symptoms and sleep quality during COVID-19 outbreak in China: a web-based crosssectional survey. Psychiatry research 288, 112954, http://dx.doi.org/10.1016/j.psychres.2020.112954 Jiao, W. Y., Wang, L. N., Liu, J., Fang, S. F., Jiao, F. Y., Pettoello-Mantovani, M., Somekh, E. (2020): Behavioral and emotional disorders in children during the COVID-19 Epidemic. The Journal of pediatrics 221, 264-266, http://dx.doi.org/10.1016/j.jpeds.2020.03.013

Mayring, P. (2015): Qualitative Inhaltsanalyse. Grundlage und Techniken. 12. Aufl. Beltz Juventa, Weinheim/Basel

Murray, L., Cooper, P. J. (1997): Editorial: Postpartum depression and child development. Psycholocigal medicine 27 (02), 253-260

National COVID-19 Science Task Force (2020): Gender aspects of COVID-19 and pandemic response. In: https://sciencetaskforce.ch/wp-content/uploads/ 2020/10/Gender-aspects-of-COVID19-and-pandemicresponse-14-May-20-EN.pdf, 29.12.2020

Rajkumar, R.P. (2020): COVID-19 and mental health: A review of the existing literature. Asian journal of psychiatry 52, http://dx.doi.org/10.1016/j.ajp.2020.102066

Rosenthal, G. (2015): Interpretative Sozialforschung. Eine Einführung. 5. Aufl. Beltz Juventa, Weinheim/ Basel

Schweizerischer Bundesrat (2020 a): Verordnung über die Abfederung der wirtschaftlichen Auswirkungen der Massnahmen zur Bekämpfung des Coronavirus (Covid-19) auf die institutionelle familienergänzende Kinderbetreuung (Covid-19-Verordnung familienergänzende Kinderbetreuung). In: https:// www.bsv.admin.ch/bsv/de/home/sozialpolitischethemen/familienpolitik/vereinbarkeit/corona-kinder betreuung.html, 29.12.2020

Schweizerischer Bundesrat (2020 b): Verordnung 2 über Massnahmen zur Bekämpfung des Coronavirus (COVID-19). In: https://www.admin.ch/opc/de/classi fied-compilation/20200744/202004020000/818.101. 24.pdf, 29.12. 2020

Shuey, E. A. (2020): Early childhood education and care in the face of coronavirus. In: https://oecdedu today.com/early-childhood-education-and-care-corona virus/?fbclid=IwAR3G6NzTiLUZXCKVjEBYRakekJSmGF 6k5AljNLN7_OwAbD2oU7ZNHyC_Ols, 28.7.2020
Stein, A., Gath, D. H., Bucher, J., Bond, A., Day, A. Cooper, P.J. (1991): The relationship between postnatal depression and mother-child interaction. The British Journal of Psychiatry 158 (1), 46-52

Sticca, F., Brauchli, V., Lannen, P. (2020): Ist es ok, wenn mein Kleinkind YouTube Videos schaut? Eine Gegenüberstellung verschiedener Empfehlungen zur Nutzung digitaler Medien in der frühen Kindheit. Frühförderung Interdisziplinär 39 (4), 225 - 227, http://dx.doi.org/10.2378/ft2020.art22d

Teti, M., Schatz, E., Liebenberg, L. (2020): Methods in the time of COVID-19: The vital role of qualitative inquiries. International Journal of Qualitative Methods 19(51), 1 -5, http://dx.doi.org/10.1177/1609406920 920962

The Alliance for Children Protection in Humanitarian Action, End Violence Against Children, UNICEF, WHO (2020): COVID-19: Protecting children for violence, abuse and neglect in the home. In: https://www.unicef.org/sites/default/files/2020-05/ COVID-19-Protecting-children-from-violence-abuseand-neglect-in-home-2020.pdf, 30.7.2020

Wang, C., Pan, R., Wan, X., Tan, Y., Xu, L., Mclntyre, R. S., Choo, F. N., Tran, B., Ho, R., Sharma, V. K., Ho, C. (2020): A longitudinal study on the mental health of general population during the COVID-19 epidemic in China. Brain, behavior, and immunity 87, 40-48, http://dx.doi.org/10.1016/j.bbi.2020.04.028

Weissbourd, R., Batanova, M., Mclntyre, J., Torres, E. (2020): How the pandemic is strengthening fathers' relationship with their children. In: https://mcc.gse. harvard.edu, 18.10.2020

Wenham, C., Smith, J., Morgan, R. (2020): COVID-19: the gendered impacts of the outbreak. The Lancet 395 (10227), 846-848, http://dx.doi.org/10.1016/ S0140-6736(20)30526-2

Wesemann, U., Bühler, A., Rose, C., Willmund, G. (2020): Momente der Wahrheit - Quarantäne bis Home office: Empfehlungen zum Erhalt der mentalen Fitness im Umgang mit COVID-19. WMM 64(S1)

Wustmann Seiler, C., Simoni, H. (2016): Orientierungsrahmen für frühkindliche Bildung, Betreuung und Erziehung in der Schweiz. Erarbeitet vom Marie Meierhofer Institut für das Kind, erstellt im Auftrag der Schweizerischen UNESCO-Kommission und des Netzwerks Kinderbetreuung Schweiz, Zürich 